\title{
Lectura y comprensión lectora mediante las tecnologías de la información y comunicación en educación básica ${ }^{1}$
}

\section{$\overline{\text { Reading and reading comprehension through information and }}$ communication technologies in basic education}

\author{
DOI: http://dx.doi.org/10.17981/cultedusoc.9.3.2018.68 \\ Artículo de investigación. Fecha de recepción: 15/06/2018. Fecha de aceptación: 27/11/2018 \\ Deniris Pulgar-Calvo'; \\ Dennys Bolaño-Pérez; Dino Martínez-Peña; \\ Fabiola De la Cruz-Torregrosa; José Rodríguez-Sierra; \\ María Martínez-Vásquez; Miladis Meza-Ramos y Mileida Castillo-Rodríguez ${ }^{3}$ \\ Institución educativa Francisco de Paula Santander, sede José Maria Córdoba (Colombia) \\ ovloc2012@hotmail.com \\ Pulgar-Calvo, D., Bolaño-Pérez, D., Martínez-Peña, D., De la Cruz-Torregrosa, F., Rodríguez-Sierra, J., Martínez-Vásquez, M., Meza- \\ Ramos, M. y Castillo-Rodríguez, M. (2018). Lectura y comprensión lectora mediante las tecnologías de la información y comunicación \\ en educación básica. Cultura. Educación y Sociedad 9(3), 567-580. DOI: http://dx.doi.org/10.17981/cultedusoc.9.3.2018.68
}

Para citar este artículo:

\section{Resumen}

La lectura juega un papel importante en el desarrollo integral de los educandos, fortaleciendo y potenciando sus capacidades de interpretación, comprensión y habilidades comunicativas básicas para un buen desempeño escolar. El estudio busco fortalecer la lectura y comprensión lectora mediante las Tecnologías de la Información y Comunicación (TIC) en educación básica. Con un diseño metodológico de tipo cualitativo bajo el modelo de investigación Acción Participativa (IAP), las técnicas de recolección de datos utilizadas fueron la observación participante y el diario de campo. Se contó con ciento veintinueve (129) participantes de la institución educativa Francisco de Paula Santander, sede José María Córdoba. Los resultados de la observación evidenciaron falencias en los procesos de lectura y comprensión lectora, omitiendo y confundiendo letras, poca fluidez verbal y desinterés por la lectura, para lo cual los docentes diseñaron estrategias pedagógicas basadas en las TIC para dar solución a dicha problemática.

Palabras clave: lectura, comprensión lectora, TIC.

\section{Abstract}

Reading plays an important role in the integral development of students, strengthening and enhancing their abilities of interpretation, understanding and basic communication skills for good school performance. The study sought to strengthen reading and reading comprehension through Information and Communication Technologies (TIC) in basic education. With a qualitative methodological design under the participatory action research (IAP) model, the data collection techniques used were the participant observation and the field diary. There were one hundred twenty-nine (129) participants from the Francisco de Paula Santander educational institution, José Maria Córdoba headquarters. The results of the observation showed weaknesses in the reading and reading comprehension processes, omitting and confusing letters, low verbal fluency and lack of interest in reading, for which the teachers designed pedagogical strategies based on ICT to solve this problem.

Keywords: Reading, Reading Comprehension, TIC.

\footnotetext{
1 Este artículo ha sido derivado del Programa de Fortalecimiento de la Cultura Ciudadana y Democrática CT+I a través de la IEP apoyada en TIC en el Departamento de Magdalena: CICLON

2 Líder del grupo de investigación "Investigadores Josema".

3 Docentes de la institución educativa Francisco de Paula Santander, sede José Maria Córdoba, del grupo de investigación "Investigadores Josema".
}

- The author; licensee Universidad de la Costa - CUC. 


\section{Introducción}

Sabemos que la comprensión lectora es una de las llaves del éxito académico y profesional de cualquier persona; sin embargo, los docentes se encuentran a menudo ante el hecho de que su alumnado no entiende lo que lee. Este problema, además, no atañe sólo a los primeros cursos de la educación Primaria, sino que se manifiesta también en los últimos cursos de la etapa, en la Educación Secundaria Obligatoria, en el Bachillerato y en la Formación Profesional.

Las habilidades lectoras se van desarrollando a medida que el individuo crece se hace necesario que en los primeros ciclos de educación se dé importancia a la comprensión por lo cual las técnicas y actividades a desarrollar, deben iniciarse desde la primera etapa escolar, con el fin de formar desde muy temprana edad el hábito de la lectura como un deleite y no como una imposición.

Es por que el objeto de estudio de este proyecto, se centra en la búsqueda de estrategias a través del uso adecuado de las Tics como herramientas facilitadoras que permitan motivar las destrezas en los niños y niñas para la lectura y comprensión lectora por medio de las diferentes herramientas tecnológicas, que permitan entonces fortalecer aprendizaje que motiven o estimulen a nuestra población hacia el hábito lector. El mundo avanza vertiginosamente, el hombre busca facilitar a través de los medios de comunicación su sistema de vida e intercambiar con el medio exterior experiencias significativas. Décadas tras décadas se han ido modernizado los equipos para ajustarlos a las diferentes necesidades tecnológicas avanzadas como la digital y otras. Estos ajustes han sido de gran utilidad, teniendo en cuenta su aplicación en cada campo: laboral, industrial, comercial y educativo.
Así entonces, las organizaciones, señalan Mendoza, López, \& Salas (2016), "son entes que generan sus propios objetivos y diseñan la forma como alcanzarlos teniendo en cuenta, necesariamente, las influencias del entorno social" (p. 62). Las escuelas no escapan de ello y es de allí que, en las organizaciones cualquiera que sea su ámbito, se hace preciso introducir una gestión estratégica como una preocupación por superarse (Mendoza, López y Salas, 2016)

Las tic se han convertido en ese impacto que ha transcendido fronteras y ha innovado en su buen uso, estrategias y alternativas que amplían las posibilidades de llegar con más eficacia a cada campo; implementando esto en el ámbito escolar y partiendo de las múltiples limitaciones que allí se encuentran entre el poco interés por la parte lectora y por ende en una buena comprensión de la misma abriendo nuevas expectativas y generando nuevos métodos y procesos que motiven y permitan elevar el hábito lector en los educandos.

Desde la observación realizada a través de la práctica pedagógica, los docentes de la institución Francisco de Paula Santander, sede José María Córdoba, identificaron que los estudiantes de primaria y segundaria presentan dificultades con las competencias lectoras, lo cual se ha convertido en una problemática puesto que se ve reflejado en el rendimiento académico de los mismos en las diferentes áreas de estudio, además, han venido presentando falencias en escritura y comprensión lectora, esto se evidencia en la omisión de letras, poca fluidez verbal, mala ortografía y caligrafía, desinterés por la lectura, pobre producción de textos. Por lo tanto, esta situación, se hace necesario implementar diversas estrategias, usando los medios de comunicación que ofrecen las TIC para crear espacios agradables, prácticos que les permita mejorar los hábitos de lectura y los niveles de comprensión, producción. 


\section{Lectura y comprensión lectora} mediante las TIC

La lectura es un proceso de naturaleza intelectual donde intervienen funciones sensoriales, psíquicas y cerebrales, que se conjugan para realizar la decodificación, comprensión e interpretación de un conjunto de signos o de un lenguaje, que podrá ser visual o gráfico (letras, ideogramas, signos), táctil (sistema Braille) o sonoro como él (código Morse) y la comprensión es una competencia lectora que consiste en interpretar y darle sentido al texto.

Las competencias esenciales para la lectura, implican la pronunciación de palabras escritas, su identificación y diferenciación, comprender su significado y entender e interpretar un texto. El hábito de la lectura es parte fundamental de la formación cultural de un individuo, pues permite desarrollar en él la capacidad de razonamiento, el sentido crítico y las competencias interpretativas, eso sin contar que contribuye a mejorar las habilidades de redacción y escritura, aumenta el léxico y estimula la imaginación (Fillola, 2010).

La comprensión lectora es un proceso más complejo que identificar palabras y significados; esta es la diferencia entre lectura y comprensión. Es una habilidad básica sobre la cual se despliega una serie de capacidades conexas: manejo de la oralidad, gusto por la lectura y pensamiento crítico. Es una tarea cognitiva que entraña gran complejidad ya que el lector no solo ha de extraer información del texto e interpretarla a partir de sus conocimientos previos (Rucoba, 2015).

La lectura comprensiva requiere que el lector sea capaz de integrar la información en unidades de sentido, en una representación del contenido del texto, que es mucho más que la suma de los significados de las palabras individuales (Estévez, 2012).
El sistema educativo no puede quedarse rezagado a las nuevas exigencias de la sociedad, es conveniente que la formación de los nuevos ciudadanos, y la apropiación de las tecnologías favorezcan el aprendizaje y así, proveer los medios y herramientas, que respalden el desarrollo de los conocimientos y de las competencias necesarias para la inserción social y la educación de calidad (Duran, 2015).

De acuerdo con Isabel Solé uno de los diversos desafíos que la escuela enfrenta es que los estudiantes asimilen el proceso de leer correctamente, este es un medio la lectura es imprescindible para moverse con autonomía en las sociedades letradas; ya que esto provoca unas desventajas en las personas que no logran este aprendizaje. Para la enseñanza de la lectura se han propuesto varios modelos con el fin de brindarles a los aprendices, herramientas para desarrollar el proceso lector. Para Isabel Solé los objetivos de la lectura son, pues, elementos que hay que tener en cuenta cuando se trata de enseñar a los niños a leer y a comprender (Solé, 2009).

Esto quiere decir que el lector interactúa con el texto dentro de un contexto y construye un determinado significado. En palabras de (Piaget, 1969) esto se llamaría proceso de adaptación, en el cual se lee, se interactúa con el texto, se traen al acto de leer la experiencia, los esquemas previos desarrollados y se relacionan con la información que se encuentra en el texto para derivar, de ahí, un significado. Es así, como de un texto se pueden encontrar diferentes lecturas; de ahí que el lector, como parte de un contexto, según el sector o al grupo social al que pertenece, puede encontrar una u otro tipo de significado en el mismo texto.

En el más reciente documento de la UNESCO, se realizan unos interesantes aportes para la enseñanza de la lectura y facilita al docente elementos para que forme a los alumnos de este siglo, donde 
el acceso a los textos, masificado por el uso de internet, ha generado lectores más críticos y con mayor avidez por la información. Y de esta forma ofrece insumos de trabajo dirigidos a hacer que los propios estudiantes tomen el comando de su enseñanza y no sean solo alumnos que reciben las lecciones. Según este documento el lector que se espera en la actualidad es aquel que participa de la comunidad letrada de forma consciente y crítica; lectores con opinión, autónomos, que sean capaces de desenvolverse de acuerdo con las exigencias del mundo actual (Lorente, 2016).

Las prácticas pedagógicas sobre la comprensión lectora deben ir en búsqueda de un lector auto regulado, es decir, aquel que logra tomar conciencia de las variables que resultan importantes para su aprendizaje, que es capaz de conocer, seleccionar, aplicar y evaluar sus propias estrategias de lectura. En este mismo orden de ideas la lectura se convierte en el núcleo fundamental del currículo ya que la lectura es base fundamental para el proceso de aprendizaje de todas las áreas y fortalece los procesos de desarrollo de una sociedad crítica, democrática y participativa, (Arceo, Rojas y González, 2001).

Según Franco, Blanco y Cortes (2013), el proceso de lectoescritura debe contemplar tres aspectos fundamentales; en primer lugar; la comprensión, que indica la forma en la que las personas interpretan y analizan el lenguaje oral y escrito, a su vez esta categoría implica habilidades como percepción del habla, conocimiento del léxico, la manera como se analiza la estructura gramatical de las oraciones y el discurso que en un texto refiere a la formulación y evaluación de conversaciones o textos extensos. En segundo lugar; la producción del habla, que indica el modo de producción de lenguaje, donde la producción busca conocer cómo se convierten los conceptos a su forma lingüística es más difícil. Y, en tercer lugar; el proceso de adquisición que indica cómo se aprende una lengua.

La articulación de la Unesco hacia la lectura está basada teniendo en cuenta la realidad cotidiana del alumno y de los distintos tipos de texto a los que el alumno se enfrenta, por lo cual el alumno debe estar capacitado para realizar una lectura comprensiva, en donde realmente se estructure la lectura desde su génesis y que el mensaje de esta realmente sea comprendido; haciendo referencia a los nuevos tipos de texto a los que el alumno debe enfrentarse, se hace necesario la extensión de estrategias que los docentes debemos trabajar para la comprensión lectora (Cardozo, 2008).

Por su parte las TIC con sus diferentes herramientas han incursionado en todos los ámbitos sociales ofreciéndole a los usuarios posibilidades inimaginables décadas atrás, de acceder a la información e interactuar con ella, llevando al ser humano a depender en muchas ocasiones de su uso, razón por la cual se hace necesario desarrollar competencias digitales como herramientas para mejorar los procesos de enseñanza orientados en la escuela. Para desarrollar aprendizajes en el aula, a partir del uso de las TIC, el docente se debe apropiar de didácticas, entendiendo esta como el sector más o menos bien delimitado del saber pedagógico que se ocupa explícitamente de la enseñanza. Con el fin de brindarle al educando una serie de posibilidades que le permitan acceder al conocimiento, especialmente si se habla de procesos de comprensión lectora (Maldonado y Clavijo, 2011).

Según Ramírez y Ampudia, (2018). La tecnología, es una herramienta que promueve y aumenta las oportunidades de los países en vías de desarrollo a través del conocimiento. En las instituciones educativas estas han tomado fuerza, puesto que la combinación de información, contexto y experiencia, permiten alcanzar 
los objetivos de las diferentes áreas de estudio, como medio para obtener y administrar el conocimiento requerido en los estudiantes.

Según Avendaño, Cortes \& Guerrero, (2015) expresan que el uso de las tecnologías de la información y la comunicación (TIC) tienen un papel importante en el desarrollo de competencias educativas y sociales, como factores predictores del desempeño académico en estudiantes de básica primaria.

Por otro lado, Herrera, (2016) menciona que las TIC se han convertido en una herramienta innovadora en el contexto educativo generando una relación ineludible que se puede aplicar a cualquier modelo pedagógico a desarrollar y a tener presente de forma trasversal como instrumento pedagógico.

Afirmando así, que las TIC, por si solas no entrarían a solucionar una problemática determinada, sino que por el contrario si no se tiene definido el objetivo de su utilización estas podrían llegar a convertirse en un agente distractor u obstáculo para los estudiantes. En la medida que los estudiantes manejen las competencias tecnológicas el docente debe estar preparado para llevarles los procesos de orientación del conocimiento en la misma forma en la que el concibe su entorno. Asociar prácticas tradicionales de la escuela con el uso del computador, permite al estudiante desde los primeros años de escolaridad, acceder, producir, comprender diferentes clases de textos en diferentes formatos y participar como agente dinamizador dentro de su proceso de formación (Maldonado y Clavijo, 2011).

Las TIC son todas las tecnologías que permiten acceder, producir, guardar, presentar y transferir información. Ellas están en todos los ámbitos de nuestras vidas, en nuestra vida social, familiar y escolar. Sus usos son ilimitados y pueden manejarse con facilidad, sin necesidad de ser un experto. Estas sirven para divertir, aprender, mantener en contacto, saber lo que está sucediendo en el mundo, expresar opiniones y conocer lo que los demás opinan. Con ellas las distancias se disminuyen, la comunicación y el intercambio de información se hace cada vez más rápido y eficientes (Villegas, 2017).

Por su parte las estrategias pedagógicas son todas las acciones realizadas por el docente, con el fin de facilitar la formación y el aprendizaje de los estudiantes. Componen los escenarios curriculares de organización de las actividades formativas y de la interacción del proceso enseñanza y aprendizaje donde se logran conocimientos, valores, prácticas, procedimientos y problemas propios del campo de formación (Bravo, 2008) Las estrategias pedagógicas suministran invaluables alternativas de formación que se desperdician por desconocimiento y por la falta de planeación pedagógica, lo que genera monotonía que influye negativamente en el aprendizaje.

El proceso de enseñanza aprendizaje se concibe como el espacio en el cual el principal protagonista es el alumno y el profesor cumple con una función de facilitador de los procesos de aprendizaje. Son los alumnos quienes construyen el conocimiento a partir de leer, de aportar sus experiencias y reflexionar sobre ellas, de intercambiar sus puntos de vista con sus compañeros y el profesor. En este espacio, se pretende que el alumno disfrute el aprendizaje y se comprometa con un aprendizaje de por vida.

En la actualidad, existen propuestas metodológicas de enfoques basados en investigación, entendidos como la forma de materializar lo que se hace y como se implementa donde surgen infinidad de propuestas metodológicas que se basan en un enfoque o toman varios para hacer de ellos una propuesta metodológica; es el caso de las pedagogías basadas o fundadas en investigación, las cuales se desa- 
rrollan en diferentes contextos y asumen variados caminos en coherencia con los paradigmas y corrientes en los cuales se inscriben. Dentro de esta teoría basada en investigación se encuentra la Enseñanza por descubrimiento y el Programa Ondas, con su propuesta de la Investigación como Estrategia Pedagógica (IEP), como una perspectiva que se desarrolla en ese enfoque y toma elementos de la pedagogía critico-liberadora y del enfoque socio cultural (Mejía y Manjarrés, 2010).

Los contenidos del proceso educativo se convierten en problemas, y a partir de preguntas se estructura el currículo, en donde los avances y desarrollos de los estudiantes se determinan por la ampliación de estructura previa que se manifiesta en lenguaje, raciocinios y conocimientos. Ese descubrimiento no es necesariamente autónomo, sino que se hace guiado por el profesor, quien se encarga de planificar los ejercicios y los procedimientos pare el fin buscado (Pozo, 2006).

El eje de la propuesta de la IEP es la idea de que en la sociedad existen saberes propios de la cultura, los cuales negocian permanentemente con las formas establecidas del conocimiento; por ello, la investigación planteada en los grupos infantiles y juveniles busca la unidad y relación de saberes y conocimientos como partes complementarias, a través de una propuesta metodológica que realiza el reconocimiento social de los actores, quienes ponen en juego esas diferentes concepciones, que podrán tramitar reconociendo la visibilidad de múltiples métodos investigativos, en coherencia con el tipo de problema.

La IEP se da una nueva constitución de subjetividad de estos tiempos, en los cuales las culturas infantiles y juveniles, en ese sentido de ser nativos digitales, no solamente hacen su uso, sino que impugnan esa desigualdad en su práctica pedagógica, interpelan la legitimidad de ella y construyen horizontes de posibilidad en donde su práctica grupal le muestra que hay futuro compartido y que él aquí y ahora, con esa colectividad con la que trabaja, lo está construyendo y le permite pensar esos cambios y esas transformaciones porque los comienza a vivir en su vida.

En la misma dinámica, el maestro y la maestra que trabajan con la IEP comprenden las posibilidades de esas transformaciones y se alimentan de ese ejercicio que realizan con sus grupos para producir los cambios y transformaciones en la esfera de su práctica pedagógica, e inician la marcha no por una ley o un mandato, sino porque encuentran allí que pueden ser educadores de otra manera, sin miedo, en búsqueda, sin certezas, pero con la compañía del grupo con el cual van reelaborando su práctica.

Ahí, en la esfera del mundo micro social van surgiendo los gérmenes de las nuevas colectividades que dan forma a la utopía de estos tiempos en educación, y él y ella participan en ellas sabiendo que es un campo en construcción, y ambos son parte de ello, haciendo real y concreta la idea de "vida buena", planteada por nuestros grupos originarios como una manera de mantener la unidad de la naturaleza y la cultura.

Por ello, la propuesta no es formar científicos, es construir una cultura ciudadana y democrática en ciencia, tecnología e innovación para estos tiempos de un mundo construido sobre el conocimiento, la tecnología, la información y la comunicación. Este ejercicio pedagógico aparece como fundamento de una nueva forma de lo público que, a través de la idea de justicia educativa y justicia curricular, trabaja por construir sociedades más justas y menos desiguales. Y si después de esto los niños, niñas y jóvenes optan por ser científicos lo serán también de otra manera, como parte de la búsqueda iniciada desde la propuesta metodológica que contiene 
como valores fundamentales esas capacidades de lo humano (cognitivas, afectivas, valorativas y de acción) sobre las cuales se fundamenta la investigación como estrategia pedagógica.

El punto de partida para buscar caminos alternativos en educación desde una perspectiva crítica se da a partir de un triple reconocimiento: Primero, de cómo, derivado de la revolución científico-técnica en marcha, se asiste a una reconfiguración del saber escolar de la modernidad, lo cual se debe convertir en una oportunidad para ir más allá de una simple modernización de la educación e intentar transformar las relaciones de poder que controlan y dominan con propuestas pedagógicas y metodológicas inclusivas que muestren diseños concretos, más allá de la simple denuncia y crítica general a esa modernización.

El segundo hecho por ser reconocido es el lugar que el trabajo inmaterial (trabajo intelectual) ha tomado en esta sociedad, al convertirse en un generador de valor y, por lo tanto, elemento central para construir el capitalismo de este tiempo, lo cual convierte a actores que trabajan con el conocimiento, la tecnología, la información y la comunicación en sujetos claves de los nuevos procesos de conformación de esta sociedad, alcanzando los educadores de todos los tipos un carácter que organiza y da forma con base y fundamento de estas modificaciones sociales (Mejía, 2011).

Es un ejercicio que va a requerir a todo educador, reconocerse y construirse como actor en estas nuevas condiciones, lo cual va a exigir un replanteamiento de las formas anteriores y la consabida modificación de su nueva condición, así como de las transformaciones institucionales en las cuales desarrolla su quehacer. En ese sentido, su subjetividad y los escenarios de su acción se convierten en campos sistematizados y por lo tanto, en disputa para construir un proyecto de emancipación o de simple modernización al servicio de los grupos dominantes en la sociedad. Es allí donde el educador pone en escena su capacidad humana al servicio de intereses precisos y concretos (Cetrulo, 2001).

El tercer elemento por reconocer desde una perspectiva crítica es cómo uno de los aspectos centrales en la configuración de un trabajo inmaterial es la investigación, la que da forma a este nuevo proyecto de control del capital, siendo este uno de los trabajos de este tiempo que más valor genera, por lo tanto, es clave en la modalidad actual de acumulación-. Allí se reconoce el lugar de ella, como un factor clave en la configuración de la revolución época en la cual nos encontramos.

A la vez que ha generado estas condiciones nuevas en la sociedad, se ha constituido a sí misma como campo de conocimiento, generando saberes y aproximaciones diversas desde y hacia ella, y en muchas ocasiones, con sus particularidades especificidades. En ese sentido, pensar la investigación desde las corrientes educativas criticas significa una lucha teóricopráctica por la manera como sus presupuestos sobre el conocimiento, la ciencia, su epistemología, la cultura, lo humano y los grupos sociales enmarcan una acción que durante cuatrocientos años ha sido señalada como objetiva, y que no solo ha construido una forma de ella, sino que también ha ayudado a generar formas de poder que en la sociedad han servido para el control y la gestación de desigualdades, y en estos tiempos, nuevas formas de acumulación y dominación.

Dicho en otras palabras, se requiere reconocer la importancia de la investigación en la sociedad, a la vez que se develan los intereses presentes. En su práctica forja una mirada crítica, que en coherencia con los tiempos presentes se ve obligada no solo a la denuncia, sino también a apropiarse de ella como una realidad muy importante en esta época para construir 
propuestas que a la vez que promuevan su apropiación práctica crítica, sirva no solo para modernizar, sino, ante todo, para transformar contextos, culturas, epistemologías, y redirigir sus escenarios de poder para construir subjetividades y ciudadanías que reconociendo el lugar de lo local se abran a un tiempo-espacio global y a las nuevas mediaciones científicotecnológicas de este tiempo para hacer concreta la construcción sociedades más democráticas, justas y humanas, así como la necesidad de un planteamiento sobre ella en cualquier proyecto emancipador.

En América Latina, así como en grupos subalternos en el mundo del Norte y en diferentes actores críticos de otros continentes, se ha desarrollado, en la perspectiva de los acumulados del paradigma latinoamericano, una concepción de trabajo educativo que se caracteriza por ser una acción política en la esfera de la educación; ella busca transformar las condiciones de control, dominio y formas de sujeción de actores, comunidades e instituciones. Para lograrlo busca generar conciencia crítica y dinámicas sociales que impulsen que los grupos construyan formas de asociación y organización que los convierte en sujetos colectivos, constructores de su historia.

La investigación como estrategia pedagógica ha tomado de la educación popular algunos de sus elementos básicos y los ha convertido en ejes de su propuesta, diferenciándose de otros acercamientos de pedagogías fundadas en la investigación, a la vez que realiza un proceso de construcción colectiva de su propuesta, donde incorpora niños, niñas, jóvenes, maestras, maestros, asesores, dándole forma a una particular manera de generar procesos de educación popular con estos grupos. Por ello su punto de partida es la realidad de estos grupos y sus saberes, para elaborarlos a partir del proceso investigativo.

En los departamentos y municipios, los actores optan por desarrollar la propues- ta; es decir, constituyen redes (sociales, políticas, de actores institucionales) que hacen real esa búsqueda y se coordinan para concretarla en los diferentes territorios y espacios de sus respectivas localidades. Estas redes han reconocido la necesidad de transformar los procesos de la educación, de la relación entre adultos y niños y niñas, así como de las relaciones sociales que se mediatizan en estas prácticas.

Por ello, esa opción se desarrolla en el contexto, con las particularidades de la cultura, desde las subjetividades constituidas. Todas ellas en una relación: organizaciones sociales, subjetividades, prácticas sociales, dinamizando un escenario de individuación, en donde la integralidad de lo humano (razón, emoción, acción, intereses) debe ponerse en juego para reconocer que nos hacemos humanos en la diferencia y en una interacción conflictiva con los otros y sus realidades.

Allí le da forma a ese otro principio de la educación popular, en la cual la realidad se conoce para transformarla transformándonos a nosotros mismos, y esto desde un horizonte ético que busca develar y enfrentar la segregación, la exclusión y formas de control y dominación que se produce por las múltiples manifestaciones del poder en nuestra sociedad: políticas, económicas, sociales, étnicas, en el conocimiento, de subjetividades, de género y en el ejercicio de la práctica educativa y pedagógica; construir un espíritu de emancipación humana, para no desarrollar procesos de poder que controlan ni dominan, ni permitir que otros lo realicen en su propia vida, (Torres, 2007).

La IEP postula la existencia de saberes que se mueven más en la experiencia y las actuaciones de los seres humanos, y de conocimientos que están más dados en la esfera de las disciplinas; de esta forma, postula la existencia de racionalidades diferentes a la euro céntrica norteameri- 
cana, las cuales también deben ser reconocidas por el ejercicio educativo, haciendo real la interculturalidad y la negociación cultural, (Mignolo W, 2003).

Este ejercicio debe darles cabida a las razones más allá de la razón universal, para hacer visible el reconocimiento del otro y de lo otro diferente a aquello que es postulado como universal, fruto de la lógica de control y de poder, visibilizar en los diferentes sus saberes, su historia, su cultura, y, cuando sea el caso, reconocerlos como epistemes negadas o invisibilidades. Va a ser el ejercicio de reconocer los contextos como lugares de saber. Por ello, busca devolver al acto educativo diferencia, heterogeneidad y multiplicidad fundadas en una diversidad cultural, social y cognitiva.

Desde ahí, lo que se construye en cualquier acción educativa son relaciones sociales, en las cuales se manifiestan bajo formas pedagógicas aquello que pensamos de la sociedad. Por eso, el papel del educador es ser enseñante - aprendiente, reconociendo la manera como se forma a la vez que va formando. En este sentido, el papel del formando es activo y también forma a sus adultos acompañantes, a la vez que va aprendiendo, gestándose unas relaciones educativas desde lo diferente en la interculturalidad (De Souza, 2001), en el respeto y el reconocimiento de los aportes de cada uno.

Por ello en la IEP, maestro y maestra se convierten en acompañantes co-investigadores, transformando su rol tradicional y reconociéndose como aprendientes en el proceso. En el caso de la IEP, se parte de la pregunta del sentido común de los niños, niñas y jóvenes para reelaborarla en la perspectiva de la educación popular, que reconoce la existencia de saberes comunes y elaborados así en la negociación con los conocimientos disciplinares, y de las maneras como se correlacionan en la negociación cultural, lo cual muestra la manera en que se van dando estas transformaciones, a través del aprendizaje colaborativo, en el cual intervienen niños y adultos. En el sentido de Vigotsky, toda función aparece en dos planos: en lo social (interpsíquico) y en lo psicológico (intrapsíquico).

La idea de aprendizaje es retomada también de este enfoque y reelaborada para Vigotsky: El aprendizaje es un aspecto universal y necesario del proceso de desarrollo culturalmente organizado y específicamente humanos de las funciones sicológicas; es decir, este, organizado pedagógicamente, se convierte en desarrollo mental y sigue generando procesos evolutivos que no se podrían dar sin él. Es allí donde las relaciones entre el sujeto y el mediador son activas y recíprocas, en la internalización se da el conocimiento. En ese sentido, el aprendizaje es una actividad propia diferenciada, por ello no se puede hacer equivalente a desarrollo.

\section{Utilización de esquemas y mapas conceptuales}

Para Sanz (2013), las estrategias empleadas para mejorar la comprensión de textos consisten en utilizar esquemas o mapas semánticos. El fundamento teórico de esta técnica se basa en las teorías cognitivas del procesamiento y almacenamiento de la información en forma de esquemas. Partiendo de la base de que la información no se almacena linealmente, sino formando estructuras que organizan la información según su importancia jerárquica, distintos autores han supuesto que al utilizar técnicas que compartiesen esos principios se facilitaría la comprensión y retención de textos, ya que se produciría un procesamiento más profundo. Una estructura de contenido de un texto presenta de forma esquemática las ideas y relaciones que aparecen en una lectura. Permite una visión global del tema y contextualiza las 
partes en un todo más general. Los autores han denominado a estas estructuras de muy distintas formas. Norman, las llama redes semánticas. Estas redes representan los nodos y sus relaciones.

\section{Metodología}

\section{Diseño}

El diseño metodológico del estudio se sustenta en el enfoque cualitativo, este se hace en el contexto natural rescatando los significados elaborados en las interacciones sociales en ella prevalece lo individual, lo colectivo en cuanto a la elaboración de significados y lo subjetivo; además es de carácter interpretativo y desarrolla mediante diseños abiertos y con técnicas poco estructuradas para dar espacio a la comprensión del sentido de la acción humana antes que la explicación y la descripción.

Además, se guio bajo el paradigma acción - participación, trata de identificar la naturaleza profunda de las realidades, su sistema de relaciones, su estructura dinámica, centrándose en la exploración, descripción, análisis e interpretación de datos ricos y profundos de la realidad de los grupos estudiados. Además, en ella existe una mayor tendencia a los procesos de comunicación entre el investigador y los investigados, mayor naturalidad y habilidad de estudiar los factores sociales en un escenario natural.

\section{Participantes}

La unidad de análisis estuvo conformada por ciento veintinueve (129) estudiantes desde el nivel Preescolar hasta el grado quinto de básica primaria de la Institución Educativa Departamental Francisco de Paula Santander Sede José María Córdoba, los participantes cumplieron con el requisito de diligenciar el consentimiento informado, los cuales fueron distribuidos en grupos de la siguiente manera (tabla 1 ).

TABLA 1

Distribución de la unidad de análisis

\begin{tabular}{ccc}
\hline $\begin{array}{c}\text { Grados y } \\
\text { grupos }\end{array}$ & $\begin{array}{c}\text { Número de } \\
\text { alumnos }\end{array}$ & Jornadas \\
\hline Preescolar & 17 & Mañana \\
Primero A & 13 & Mañana \\
Primero B & 14 & Mañana \\
Primero C & 16 & Mañana \\
Segundo & 17 & Mañana \\
Tercero A & 12 & Tarde \\
Tercero B & 11 & Tarde \\
Cuarto & 17 & Tarde \\
Quinto & 12 & Tarde \\
& Total & 129 \\
\hline
\end{tabular}

Fuente: elaboración propia.

\section{Instrumentos y técnicas}

En cuanto a los instrumentos de recolección se utilizó la observación y el diario de campo, la observación es un instrumento que ayuda al investigador a inmiscuirse de forma total en el ambiente con el cual se va a encontrar, es un proceso de hechos, recolección de datos y análisis, donde el investigador entiende a los participantes y no se dedica únicamente a registrar hechos. El diario de campo es parte de un registro cronológico que se desarrolla mediante la sesión compartida con los infantes, es donde se describe detalladamente los aspectos positivos como negativos colocando significado a cada uno de ellos como un recurso de mejoras en todas las áreas con el grupo a trabajar considerando los recursos manejados y utilizados. Un cuaderno donde se va a descubrir la manera de expresar y escribir frente a la relación que se tiene con los infantes, es un momento de no cohibir lo vivido y las experiencias obtenidas. 


\section{Procedimiento}

La metodología del proyecto de investigación se realizó a partir de los recorridos de las trayectorias de indagación, basados en la integración de la IEP apoyada en TIC al aula, articulado al plan de estudios, transversalizando así los procesos de lectura y comprensión lectora a todas las áreas del conocimiento, lo anterior con la finalidad de generar una trasformación social y educativa a través del aprendizaje en contexto, involucrando intereses, motivaciones y realidades de los estudiantes para generar conocimiento científico, donde se establecieron cinco (5) etapas que especifican la ejecución y las metas trazadas para darle solución a la pregunta problema planteada. Las etapas utilizadas se presentan a continuación.

- Etapa (1): Convocatoria y acompañamiento para la conformación de los grupos de investigación; en esta etapa se seleccionó la muestra para realizar el estudio y posteriormente se realizó la formulación de la pregunta y planteamiento del problema de investigación.

- Etapa (2): Identificación y definición de la temática de investigación, en esta etapa se realizó la fundamentación teórica.

- Etapa (3): Diseño de las trayectorias de indagación, donde se ejecutó la organización del proceso de sistematización, se seleccionaron los instrumentos de recolección de información, se estructuro el diseño metodológico y el proceso de recolección y registro de información.

- Etapa (4): Producción de saber y conocimiento, esta consistió en el análisis de información y redacción del informe de sistematización.
- Etapa (5): Apropiación social del conocimiento, se realizó la divulgación de resultados de investigación en Espacios de apropiación municipal "El maestro tiene la palabra".

\section{Resultados}

De acuerdo a los hallazgos encontrados en el proceso de investigación a partir de la observación inicial se evidenciaron falencias en los procesos de lectura y comprensión lectora, omitiendo y confundiendo letras, poca fluidez verbal y desinterés por la lectura, al indagar por las causas de estas falencias se encontró; que los padres dedican poco tiempo a sus hijos, hay falta de material de lectura, no les gusta leer y por lo tanto no comprenden, y también cuentan con atención dispersa por lo cual se desconcentran con otros estímulos del medio.

A partir del análisis de las técnicas e instrumentos que se utilizaron en los estudiantes de la sede" José María Córdoba" se evidencio que es importante hacer un diagnóstico apropiado del nivel de comprensión lectora que maneja el estudiante antes de aplicar cualquier estrategia que pretenda mejorar la capacidad para interpretar textos, pues de esta manera se logra hacer un seguimiento de los avances del educando y asociarlo con conocimientos previos a la lectura.

Mediante las observaciones registradas los docentes diseñaron estrategias basadas en la implementación de las TIC, puesto que, por su carácter innovador los estudiantes se ven motivados e interesados en utilizar las herramientas, favoreciendo la comprensión de lo aprendido, debido a que dichas estrategias tienen en cuenta los estilos de aprendizaje y favorecen el proceso de aprendizaje. Las actividades diseñadas se plantearon de acuerdo al grado escolar, las cuales se definen a continuación (tabla 2) 


\section{Tabla 2}

Actividades pedagógicas y recursos para la implementación de las TIC según grado escolar

\begin{tabular}{cll}
\hline Grado & Actividades & Recursos \\
Preescolar & $\begin{array}{l}\text { Cuentos infantiles. Videos } \\
\text { didácticos }\end{array}$ & $\begin{array}{l}\text { Televisor. Parlantes, memoria USB, } \\
\text { pizarrón, marcadores, videos, copias para } \\
\text { la realización de talleres. }\end{array}$ \\
Primero & Video “El Mono Sílabos” & $\begin{array}{l}\text { Portátil, video beam, pizarrón, } \\
\text { marcadores, televisor, videos, copias para }\end{array}$ \\
& & la realización de talleres. \\
Segundo & Videos sobre temáticas de & $\begin{array}{l}\text { Portátil, video beam, pizarrón, } \\
\text { marcadores, televisor, videos, copias para }\end{array}$ \\
Tercero & Juegos interactivos & la realización de talleres. \\
Cuarto & ¡Qué divertidos es leer! & Tablets y conectividad a internet. \\
Quinto & Explorando en internet & Tablets y conectividad a internet. \\
\hline
\end{tabular}

Fuente: elaboración propia.

\section{Conclusiones}

Las TIC son herramientas que han incursionado en todos los ámbitos sociales ofreciéndole a los usuarios posibilidades de acceder a la información e interactuar con ella, llevando al ser humano a depender en muchas ocasiones de su uso, razón por la cual se hizo necesario desarrollar competencias digitales para mejorar los procesos de enseñanza orientados en la escuela. La estrategia fue didáctica y de carácter constructivista aplicada a los estudiantes, donde las actividades desarrolladas con los estudiantes contaron con el uso de material impreso y digital lo cual posibilitó un mejor acercamiento a la información.

Desde las reflexiones realizadas sobre el proceso de investigación se recomienda brindar mayor capacitación a los docentes de básica primaria para que trabajen desde el aula con herramientas TIC y realizar una reestructuración curricular en el área de lenguaje, con el fin de buscar que los profesores aprovechen plenamente las oportunidades de desarrollo profesional en tecnologías.

Integrar sistemáticamente el uso de las TIC al currículo, en especial en el área del lenguaje para mejorar el nivel crítico de la comprensión lectora en los estudiantes mediante la activación de sus conocimientos previos. La participación de los estudiantes en el desarrollo de la estrategia didáctica mediada por las TIC, generó en ellos su vinculación activa al proceso de aprendizaje, reconociendo sus fortalezas y debilidades frente a la comprensión lectora.

Es evidente la mejoría que registran los niveles de comprensión lectora en los estudiantes del grupo. El trabajo en el aula permitió el aprendizaje colaborativo, permitiendo la retroalimentación permanente como parte del proceso de comprensión de lectura, así como también el dialogo de saberes que muchas veces no se generaba en los estudiantes, negándose emitir sus opiniones por miedo al rechazo o a equivocarse. 


\section{Referencias}

Arceo, F., Rojas, G. y González, E. (2001). Estrategias docentes para un aprendizaje significativo: una interpretación constructivista. McGraw-Hill.

Avendaño, I., Cortés, O. y Guerrero, H. (2015). Competencias sociales y tecnologías de la información y la comunicación como factores asociados al desempeño en estudiantes de básica primaria con experiencia de desplazamiento forzado. Diversitas, 11(1), 1336. Recuperado de http://www.redalyc. org/articulo.oa?id=67943296001

Bravo, H. (2008). Estrategias pedagógicas. Córdoba: Universidad del Sinú.

Cardozo, J. (2008). Estrategias metodológicas para fomentar la lectura en niños y niñas de 5 a 6 años, en el CED Buenavista. Universidad de San Buenaventura, Bogotá, D.C.

Cetrulo, R. (2001). Alternativas para una acción transformadora: Educación popular, ciencias y política. Montevideo: Trilce-Instituto del Hombre.

Durán, C. (2015). Relevancia y pertinencia didáctica de los medios tecnológicos en la enseñanza de las ciencias. [Maestría]. Unicach, Tuxtla Gutiérrez. Recuperado de https://repositorio.unicach.mx/handle/20. 500.12114/205

Estévez, I. (2012). Estrategias de aprendizaje para la comprensión lectora en inglés para estudiantes de primer año medicina. Gaceta Médica Espirituana, 13(2), 6 .

Fillola, A. (2010). Función de la literatura infantil y juvenil en la formación de la competencia literaria/Antonio Mendoza Fillola. Alicante: Biblioteca Virtual Miguel de Cervantes. Recuperado de http://www.cervantesvirtual.com/nd/ark:/59851/bmc5h7z4
Franco, M., Blanco, P. y Cortés, O. (2013). Papel de las habilidades metalingüísticas en los procesos de lectura y escritura en la educación superior. Escenarios, 11(2), 82-86.

Herrera, B. (2016). Cultura Ciudadana y las Tecnologías de la Información y la Comunicación. Barranquilla: Yoyobiz. .

Lorente, L. (2016). La educación en América Latina hoy en el horizonte de la agenda educativa post 2015. Revista Española de Educación Comparada, (27), 17-34.

Mejía, M. y Manjarrés, M. (2010). La Investigación como Estrategia Pedagógica. Bogotá, D.C.: Programa Ondas - Colciencias.

Mejía, M. (2011). La Sistematización. Una forma de investigar las prácticas. Lima: Fondo Editorial del Pedagógico San Marcos.

MEN. (1998). Competencia lectora. Obtenido de http://www.mineducacion.gov. co/portal/

Mendoza, D., López, D. y Salas, E. (2016). Planificación estratégica de recursos humanos: efectiva forma de identificar necesidades de personal. Económicas CUC, 37(1), 61-79. http://dx.doi.org/ 10.17981/econcuc.37.1.2016.03

Mignolo, W. (2003). Capitalismo y geopolítica del conocimiento. Buenos Aires: Paidós.

Pacheco, C. (2013). Estrategias empresariales más utilizadas por las pymes en Sincelejo. Económicas CUC, 34(1), 183-202. Recuperado de https://revistascientificas.cuc.edu.co/economicascuc/article/view/583/pdf_75

Piaget, J. (1969). Psicología y Pedagogía. Barcelona: Ariel Editores.

Pozo, J. y Gómez, M. (2006) Aprender y enseñar ciencia. Madrid: Morata. 
Ramírez, R. y Ampudia, D. (2018). Factores de Competitividad Empresarial en el Sector Comercial. Revista Electrónica de Ciencia y Tecnología del Instituto Universitario de Tecnología de Maracaibo (RECITIUTM). 4(1). 1632.

Rucoba, J. (2015). Comprensión Lectora y sus efectos en el aprendizaje de la lectoescritura en los estudiantes del Primer Grado de la Institución Educativa Pública. Juliaca.

Saez, A. (2013). Como Diseñar actividades de comprensión lectora. Navarra: Departamento de Educación.

Solé, I. (2009). Estrategias de lectura. Barcelona: Grao.
Strack, J., Suárez, J., Di Mauro, G. y Jacob, S. (2014). Impacto de la iluminación residencial eficiente en la calidad de la energía de una red de distribución. INGE CUC, 10(2), 9 - 19. Recuperado de https:// revistascientificas.cuc.edu.co/index.php/ ingecuc/article/view/485

Torres, A. (2007). La educación popular. Trayectoria y actualidad. Bogotá, D.C.: El Búho.

Villegas, Y. (2017). Diseño y producción de una Revista Digital Institucional para la Escuela Normal Superior De Ocaña, Norte De Santander. [Doctoral dissertation]. Universidad Francisco de Paula Santander Ocaña, Ocaña, Colombia. Recuperado de http://repositorio.ufpso.edu. c o: $8080 / \mathrm{d} \mathrm{s} \mathrm{p} \mathrm{a} \mathrm{c} \mathrm{e} \mathrm{u} \mathrm{f} \mathrm{p} \mathrm{s} \mathrm{o} \mathrm{/} \mathrm{b} \mathrm{it} \mathrm{s} \mathrm{-}$ tream/123456789/1764/1/30737.pdf 